\title{
Residual Losses in a Guard-Ring Micrometer-Electrode Holder for Solid-Disk Dielectric Specimens
}

\author{
Arnold H. Scott and William P. Harris
}

(Januáry 19, 1961)

\begin{abstract}
A guard-ring micrometer-electrode holder especially designed for use in making dielectric measurements on solid-disk specimens of low-loss materials has been constructed. A method of determining the separation of the electrodes to \pm 1 micron, using ball reference gages, is described. Residual losses in the guard-ring holder, and also in the bridge standard capacitor used in the Schering bridge employed for the measurements, caused by losses in surface films on the electrodes and by series resistance in the leads, were accurately measured. It is shown that at frequencies from 100 cycles per second to 100 kilocycles per second these residual losses are not more than a few microradians. Using corrections thus obtained, the holder-bridge combination can be used to measure the losses of low-loss materials with greater accuracy than heretofore.
\end{abstract}

\section{Introduction}

This paper describes a guard-ring micrometerelectrode holder and methods by which the separation of the electrodes, the capacitance, and the residual losses of the holder can be precisely determined. The holder is one which was designed and constructed to meet the need for a very accurate method of measuring the dielectric properties of some standard reference specimens of dielectrics.

Such standard reference specimens $[1]^{1}$ are useful in checking the various techniques and equipment used for measurements of the dielectric constant and dissipation factor of insulating materials. Two general classes of dielectric measurements are used: two terminal and three terminal. The three terminal method is usually more accurate, but may require more time, especially if a three-terminal bridge with an earthing circuit is used. For routine measurements in quantity, the two terminal method is often used. This method generally requires corrections for the edge capacitance and ground capacitance of the specimen under test. Standard dielectric reference materials permit a check on the accuracy of such measurements.

Recently there have come into general use twoterminal holders which eliminate most of the edge capacitance and all of the ground capacitance from the measurement, and permit relatively rapid measurements to be made. However, the loss measurements include the loss over the edge of the specimen and the loss through the insulation of the highpotential electrode of the holder. Accurate determination of the electrode spacing is also required. Here again, standard dielectric reference specimens are useful in checking the techniques and measurements involved.

In order for standard reference specimens to be useful, it is necessary that their properties be known

\footnotetext{
1 Figures in brackets indicate the literature references at the end of this paper.
}

accurately, apart from the methods and equipment used to measure these properties. This requires that residual losses and capacitances in the specimen holder be reduced to negligible proportions, or if this is not feasible, that they be accurately determined and separated from the measurements made on the specimens. A further complication arises from the fact that most materials that might be used for standard reference specimens exhibit properties that are affected by the ambient humidity, and must therefore be studied under different conditions of humidity in order that this effect might be evaluated, and that the dielectric properties under a given set of conditions might be known. This involves the determination of the variation of the residuals as a function of humidity. Of course, temperature dependence must also be considered.

The foregoing considerations prompted the construction of the specimen holder to be described, and led to the measurements made to determine the residual losses under various conditions. Methods are described which permit the determination of the losses in the holder, arising from series resistance in the connecting leads, from losses inherent in any film that may exist on the surfaces of the electrodes, or from any other source. From this, corrections can be applied to measurements made on specimens placed in the holder.

A useful bonus of the method used was that the losses in the standard bridge capacitor used were also determined, and a correction for this source of error was obtained.

\section{Description of the Specimen Holder}

The holder consists of a heavy cylindrical case with the electrodes mounted coaxially inside. The central guide rod of the movable electrode extends through the top of the case and its position is controlled by a thin nut located just above the top of the case. The position of the movable electrode is 
sensed by a micrometer which comes in contact with the top of the guide rod. Figure 1 is a cross sectional diagram of the holder, and figure 2 shows the holder installed in a controlled-humidity chamber.

The guarded electrode and guard-ring are mounted on a ceramic disk. This disk was ground to nearly optical flatness and fits into a machined recess in the base of the case structure. A hole was ground in the center of this ceramic disk so that the centering rod of the guarded electrode would fit snugly into this hole thus holding the guarded electrode accurately centered on the ceramic disk. The guarded electrode is held snugly against the ceramic disk by means of screws in recessed holes in the ceramic disk. The guard-ring was recessed to fit snugly over the outside edge of the ceramic disk so that the guardring is also accurately centered on the ceramic disk. This permits the maintenance of a uniform gap between the guarded electrode and the guard-ring. Screws through the base plate and the ceramic disk into the guard-ring hold this electrode assembly rigidly against the base plate and make the necessary electrical connection between the guard-ring and the surrounding case assembly. The surface of this electrode assembly was ground optically flat.

The upper movable electrode is also mounted on a ceramic disk whose surfaces have been accurately ground. This ceramic disk is in turn mounted on the carefully machined surface of a metal disk attached to a guide rod which extends through the top of the barrel assembly. This guide rod passes through two guide bearings about $7 \mathrm{~cm}$ apart. A metal bellows is attached to the mounting disk and to a collar which fits over the guide bearing assembly.

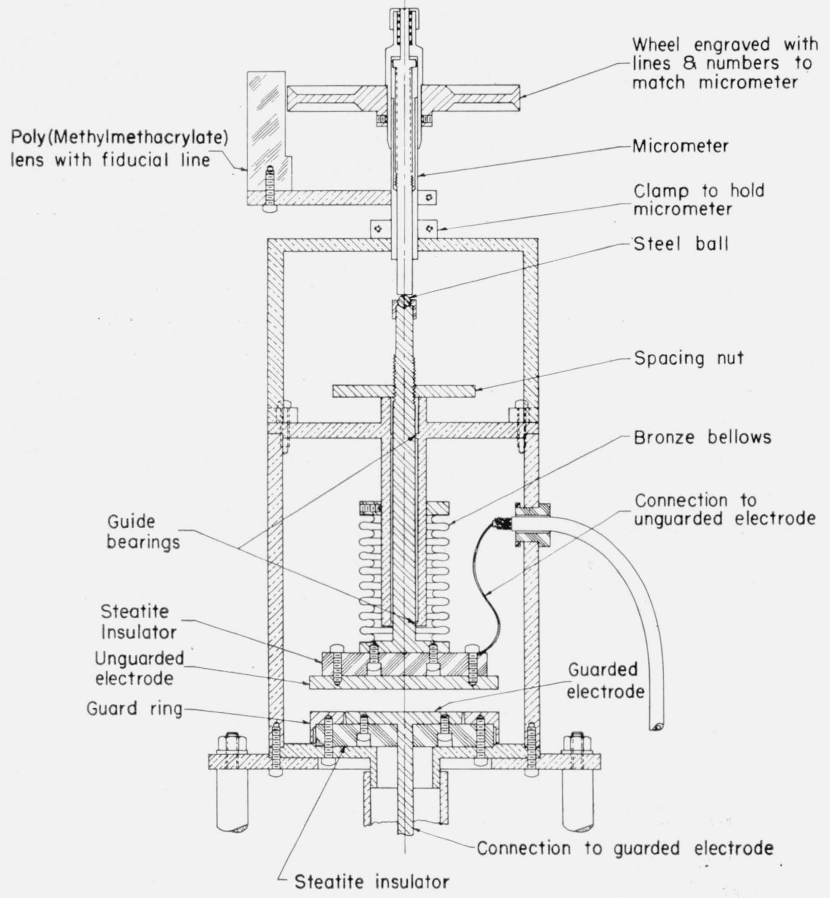

FIGURE 1. Schematic diagram of the guard-ring micrometerelectrode holder for solid-disk dielectric specimens.

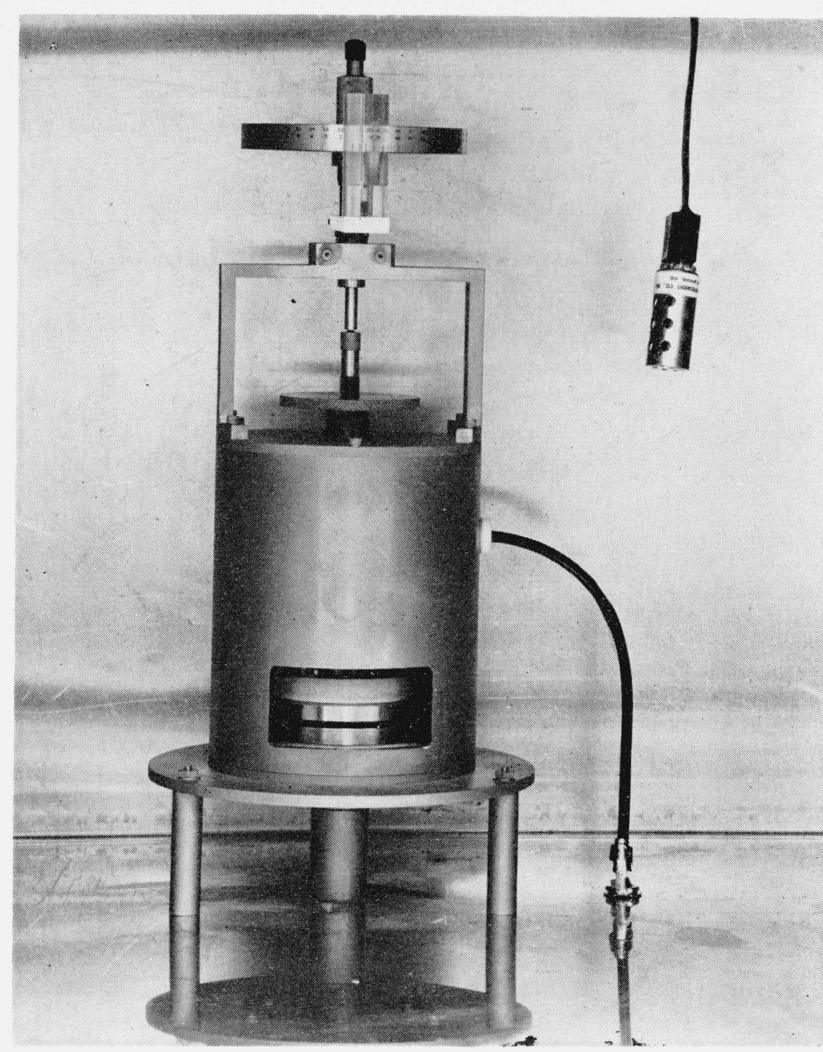

Figure 2. Photograph of the new holder in place in controlledatmosphere chamber.

A set screw in the collar permits the location of the collar so that the bellows can be made to apply a force tending to move the movable electrode toward the guard-ring assembly. The collar and bellows also prevent the rotation of the movable electrode assembly.

The upper end of the guide rod is threaded, and a. large diameter knurled nut is used on this to set the spacing between the electrodes. This nut works against the top of the guide bearing assembly. Fine threads (32 threads per inch) are used so that with care the spacing between the electrodes can be set within $1 \mu$.

The spacing between the electrodes is determined by means of a micrometer which is mounted on a frame so that it is held directly above the end of the guide rod. A steel ball is mounted on the end of the guide rod and the position of the guide rod is determined by screwing the micrometer down until it just touches this ball.

Contact with the ball can be accurately determined by a very light touch. The micrometer is equipped with a large graduated wheel so that the smallest divisions are equivalent to $2 \mu$. With a little practice and skill the micrometer measurements can be made repeatedly within a micron, by different observers.

The top and bottom of the cylindrical case can be removed to expose the electrode surfaces. The ends of the cylindrical case are carefully machined to be accurately perpendicular to the axis of the cylinder. 
The end plates and electrode assemblies are carefully ground and machined so that when they are placed on the end of the cylindrical case the electrode surfaces are also perpendicular to the axis of the cylinder. Only slight adjustments are necessary to make the electrode surfaces parallel to the accuracy which is required in this work.

The electrodes were made of A.I.S.I. Type 304 stainless steel. No plating or coating was applied. The surfaces were ground and lapped smooth.

Connection is made to the guarded electrode by means of a coaxial connector whose outer case is attached to the base plate of the holder. Connection is made to the upper electrode by a flexible wire which is clamped under one of the mounting screws which hold the electrode to the ceramic disk. The other end is connected to the core of the coaxial cable which passes through the wall of the cylindrical case.

Two windows about $7-\mathrm{cm}$ wide and $3-\mathrm{cm}$ high are cut in opposite sides of the cylindrical case at the electrode level to permit the introduction and removal of samples from between the electrodes. A sliding band around the cylindrical case can be used to cover these openings if complete shielding is required.

\section{Determination of the Area of the Guarded Electrode}

To determine the area of the guarded electrode the base plate and guarded electrode assembly were removed from the cylindrical case. The diameter of the guarded electrode, and the gap between the guarded electrode and the guard-ring were measured by means of a traveling microscope. The effective area of the guarded electrode was then computed using the gap corrections published in ASTM D-150 [2]. The effective area was found to be $20.366 \mathrm{~cm}^{2}$.

\section{Determination of the True Spacing of the Electrodes}

In order to adjust the upper and lower electrodes to be parallel and to measure the actual separation of the electrodes, a set of ball reference gages was prepared and accurately measured. Steel balls with nominal diameters of $3 / 32,1 / 8,5 / 32,3 / 16,7 / 32$, and $1 / 4$ in. were rigidly mounted in strips of plastic. These strips served as handles for placing the gages at the desired points between the electrodes, and the rigid mounting insured that a definite diameter was always presented. These reference gages were measured by the Engineering Metrology Section of NBS, and their diameters determined under no-load condition with an accuracy of $\pm 0.00005 \mathrm{~cm}$.

Parallelism of the electrodes was established by using a ball mounted similarly to those described, but not necessarily accurately measured, to complete the electrical circuit of an ohmmeter connected to the two electrodes. The electrodes were brought together by the thumb nut to such a distance that the ball just made contact between the electrode surfaces, but yet was free enough to move about.
If there was any lack of parallelism, this was made readily apparent by the existence of areas on one side of the electrodes where contact was not made while at the opposite side contact was made, or possibly the ball would not slide easily between the electrodes. Adjustments were made by tightening or loosening the appropriate screws located around the circumference of the case.

It was found that after such adjustments had been made, their full effect was not immediately seen. It took several hours for the dimensions to stabilize. This is attributed to two effects. First, the act of making the adjustment requires some handling of the cell, with some disturbance of the temperatures of the various parts of the cell case. The other factor, and perhaps the major contributor to the long time required for dimensional stability to be reestablished, is that the metal probably creeps for a time after the stresses are altered. The result of these effects is that the final adjustments must be made by making very small changes and then waiting several hours or overnight for the ensuing changes in the metal to take place.

This same ball-gage technique can be used to determine whether the guard ring and the guarded electrode are in the same plane, using the other electrode as a reference surface.

After the electrodes had been made parallel, a comparison was made between the measured capacitance and that computed for the separation of the electrodes as determined by ball reference gages of different diameters. Typical results are shown in table 1. It was concluded, from the fact that

TABLE 1. Comparison of measured capacitance with the capacitance computed from the electrode separation

\begin{tabular}{c|c|c|c}
\hline \hline $\begin{array}{c}\text { Spacing by } \\
\text { ball gage }\end{array}$ & Computed C & Measured C & $\Delta \mathrm{C}$ \\
\cline { 2 - 3 }$c m$ & $p f$ & $p f$ & \\
\cline { 2 - 3 } 0.31773 & 5.6754 & 5.6755 & 0.0001 \\
.47638 & 3.7853 & 3.7856 & .0003 \\
.55558 & 3.2457 & 3.2456 & .0001 \\
\hline
\end{tabular}

excellent agreement was obtained, that the reference gages could now be used for occasional check measurements, the measured capacitance used to determine the electrode separation with the holder empty, and these measurements used to find the zero correction to be applied to the micrometer readings. It is desirable to have a rapid means of determining this micrometer zero correction, because any adjustments of the micrometer mount, or of the electrodes, such as resetting them to be parallel, changes the micrometer zero correction. ${ }^{2}$ It was found that the zero correction can be determined readily and repeatably from capacitance measurements. Some results of such measurements are shown in table 2 .

The agreement of the micrometer zero correction determined for the various spacings is acceptable,

${ }^{2}$ A significant change of temperature could also change the correction. For this work, temperature is held constant to \pm 1 degree $\mathrm{C}$. The effect of this small temperature change was found to be negligible. 
TABLE 2. Determination of micrometer zero correction from measured capacitance

\begin{tabular}{c|c|c|c}
\hline \hline $\begin{array}{c}\text { Micrometer } \\
\text { reading }\end{array}$ & Capacitance & $\begin{array}{c}\text { Computed } \\
\text { spacing }\end{array}$ & Difference \\
\cline { 1 - 2 } $\boldsymbol{c m}$ & $p f$ & $c m$ & $c m$ \\
0.2499 & 7.177 & 0.2513 & 0.0014 \\
.1194 & 14.926 & .1208 & .0014 \\
.0793 & 22.324 & .0808 & .0015 \\
.0590 & 29.847 & .0604 & .0014 \\
.0471 & 37.159 & .0485 & .0014 \\
.0390 & 44.566 & .0405 & .0015 \\
.0332 & 52.077 & .0346 & .0014 \\
& & & \\
\hline
\end{tabular}

and following these measurements a zero correction of $14 \mu$ was used until further adjustments were made to the electrodes.

It is estimated that the separation of the electrodes can be determined to about $\pm 1 \mu$, using the micrometer in this fashion.

\section{Losses in the Specimen Holder and Standard Bridge Capacitor}

\subsection{General Considerations}

It was intended that this holder should be used during accurate measurements of the losses in materials that have rather small losses. Therefore it was necessary to evaluate the holder for the effects of inherent losses. It is evident that any series resistance in the leads connecting the holder into the measuring circuit will appear in the measurements as a loss. Also, losses in any surface films on the electrodes must be evaluated. It is known that some electrode materials form oxide films which might be expected to exhibit losses $[3,4,5,6,7,8,9$, $10,13]$.

\subsection{The Bridge}

A low-voltage Conjugate Schering Bridge was used to make the measurements. The substitution method was employed. A diagram of the bridge circuit is shown in figure 3 . Because the residual losses in the variable bridge standard capacitor $\mathrm{C}_{2}$ are involved in the measurements, it is necessary to evaluate these losses. This requirement was no appreciable handicap, because the method employed yielded simultaneously the losses in both the specimen holder and the standard bridge capacitor.

Measurements by the substitution method are made by setting $\mathrm{C}_{H}$ to some value of capacitance by adjusting the separation of the electrodes, and setting $C_{2}$, the standard bridge capacitor, to some initial value, usually $20.000 \mathrm{pf}$. The capacitor $C_{3}$ is also set to some convenient initial value. After setting Sw 1 to position $\mathrm{A}$, the bridge is balanced by varying $C_{1}, C_{4}, C_{5}$, and $R_{5}$, while alternately setting switch 2 to the $\mathrm{A}$ and $\mathrm{B}$ positions, until the detector shows a null in both $\mathrm{A}$ and $\mathrm{B}$ positions. The values of $C_{2}$ and $C_{3}$ are noted, and will be designated $C_{2}^{\prime}$ and $C_{3}^{\prime}$, respectively. Sw 1 is now moved to position $B$. Leaving $C_{1}$ and $C_{4}$ unchanged, the bridge is rebalanced by varying $C_{2}, C_{3}, C_{5}$, and $R_{5}$. The new values of $C_{2}$ and $C_{3}$ are noted, and will be designated as $C_{2}^{\prime \prime}$ and

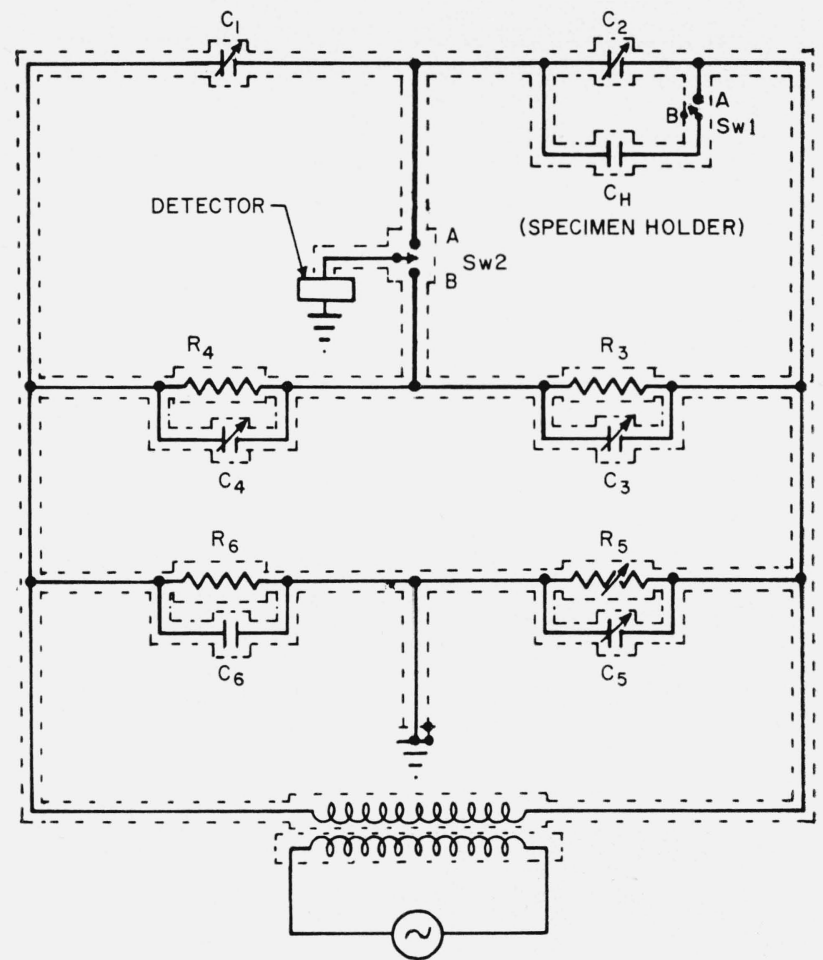

FIGURE 3. Schematic diagram of the conjugate Schering bridae used for this investigation.

$C_{3}^{\prime \prime}$. The difference between the second and first values of $C_{2}, \Delta C_{2}$, is the capacitance of the holder, ${ }^{3}$ $C_{H}$. The difference between the second and first values of $C_{3}, \Delta C_{3}$ is used to compute the value of the dissipation factor of the holder, $D_{H}$, according to the equation ${ }^{4}$

$$
D_{H}=\frac{\omega R_{3} C_{2}^{\prime \prime} \Delta C_{3}}{\Delta C_{2}}+\frac{G_{2}^{\prime \prime}-G_{2}^{\prime}}{\omega \Delta C_{2}}
$$

where

$D_{H}=$ dissipation factor $(\tan \delta)$ of the specimen holder

$R_{3}=$ value of $R_{3}$, figure 3 , in ohms;

$C_{2}^{\prime \prime}=$ capacitance of $C_{2}$ with $\mathrm{Sw} 1$ in position $\mathrm{B}$, which is the total capacitance in the measuring arm of the bridge;

$\Delta C_{3}=C_{3}^{\prime \prime}-C_{3}^{\prime \prime}=$ difference between final and initial values of $C_{3}$;

$\Delta C_{2}=C_{2}^{\prime \prime}-C_{2}^{\prime}=$ difference between final and initial values of $C_{2}$;

$G_{2}^{\prime}=$ equivalent parallel conductance of $C_{2}$ corresponding to the initial value $C_{2}^{\prime}$; and

$G_{2}^{\prime \prime}=$ equivalent parallel conductance of $C_{2}$ corresponding to $C_{2}^{\prime \prime}$.

The first term on the right in eq (5.1), $\omega R_{3} C_{2}{ }^{\prime \prime} \Delta C_{3} /$ $\Delta C_{2}$ is the dissipation factor computed without re-

3 This statement is true for the case at hand, where the correction terms in the exact expression are negligible. The exact form is given in the appendix, section 7 . ${ }_{4}^{4}$ This equation is derived in the appendix, section 7 , and appears there as eq (7.7). 
gard to the second term, which is a correction term taking into account the effect of non-zero and nonconstant dissipation factor, $D_{2}$, in the bridge standard capacitor, $C_{2}$. Let us then write

where

$$
D_{H}=D_{M}+D_{2},
$$

$$
D_{M}=\omega R_{3} C_{2}^{\prime \prime} \Delta C_{3} / \Delta C_{2}=\text { the measured or appar- }
$$
ent dissipation factor as given by the uncorrected bridge measure-

and ment.

$$
\begin{aligned}
D_{2}=\left(G_{2}^{\prime \prime}-G_{2}^{\prime}\right) / \omega \Delta C_{2}= & \text { the correction term to be } \\
& \text { applied to the apparent } \\
& \text { dissipation factor to } \\
& \text { take into account the } \\
& \text { dissipation factor of the } \\
& \text { bridge standard capac- } \\
& \text { itor, } C_{2} \text {. }
\end{aligned}
$$

In order to consider the effect of elements that behave as series resistances, it will be more convenient to have eq (5.2) written in its series equivalent form, neglecting for the moment elements that behave as parallel conductances. If we separate the series resistance associated with the bridge standard capacitor, $C_{2}$, into two parts, one part corresponding to the series ohmic resistance of the leads to $C_{2}$, the other part corresponding to the losses caused by a surface film on the electrodes of $C_{2}$, eq $(5.2)$ becomes $^{5}$

$$
D_{H}=D_{M}+2 \omega R_{2 S L} C_{2}^{\prime}+\omega R_{2 S L} \Delta C_{2}+\omega C_{2}^{\prime} R_{2 S F^{\prime}}
$$

where

$R_{2 S L}=$ the equivalent series resistance of the ohmic resistance of the leads, connecand tions, etc., of $C_{2}$,

$R_{2 S F}=$ the equivalent series resistance of the film on the surfaces of the electrodes of $C_{2}$.

\subsection{Consequences of Equation 5.3}

Examination of eq (5.3) reveals that there are two correction terms involving $R_{2 S L}$, one dependent on $C_{2}{ }^{\prime}$, the initial value of the bridge standard capacitor, the other dependent on $\Delta C_{2}$. There is one term involving $R_{2 S F}$.

Let us now consider the behavior of these correction terms as a function of the capacitance of $C_{2}$, and examine their effect on the value of $D_{M}$, the uncorrected or measured value of $D_{H}$. In the following arguments, it will be assumed that all measurements are made at a fixed frequency, except as stated. Also, for any single set of curves, it will be assumed that $C_{2}^{\prime}$ has a single value, usually, in the actual measurements to be presented later, $20.000 \mathrm{pf}$.

\footnotetext{
${ }^{5}$ This relation is derived in full in the appendix, section 7 .
}

Taking first the simplest case, involving only the term due to losses in a surface film, assuming for the moment that $R_{2 S L}$ is negligible, we see that the correction term is

$$
\omega R_{2 S F}{ }^{\prime} C_{2}^{\prime}=D_{2 F}
$$

This term is a constant, even for different values of $C_{2}^{\prime}$, because $R_{2 S F}^{\prime}$ is inversely proportional to the capacitance, ${ }^{6}$ in this case $C_{2}^{\prime}$. Therefore we may plot this term versus capacitance as a straight line of zero slope, as shown in figure 4.

If we now consider the effect of a lossy surface film on the holder electrodes, where the area is constant and the capacitance is a function of the separation of the electrodes, we see that a dissipation factor proportional to the capacitance results. ${ }^{7}$ Let us designate this component of dissipation factor, due to a lossy surface film in the holder, as

$$
D_{H F}=\omega R_{H S F} C_{H}=\omega R_{H S F} \Delta C_{2},
$$

where $R_{H S F}$ is the equivalent series resistance corresponding to the surface film losses in the holder. A plot of $D_{H F}$ versus capacitance would be a straight line with positive slope, passing through $D=0$, $\Delta C_{2}=0$ (or nearly so, depending on the ground capacitances $[11,12])$. This is shown in figure 4,

From eq (5.2), we see than $D_{M}=D_{H}-D_{2}$, or in this case $D_{M}=D_{H F}-D_{2 F} . \quad D_{M}$ is plotted in figure 4, where it is seen that it is a straight line with the same slope as the plot of $D_{H F}$, and with a negative intercept equal in magnitude to the constant value of $D_{2 F}$, if the previously assumed conditions exist.

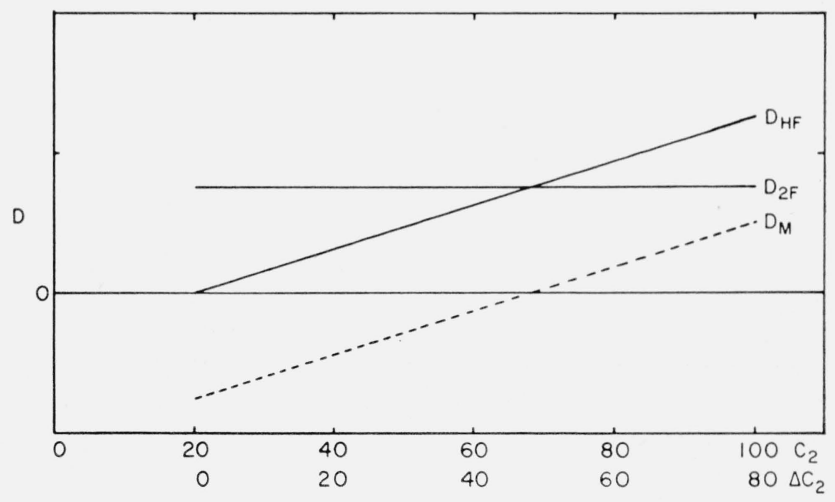

Figure 4. A plot of assumed, arbitrary values of dissipation factor due to a lossy surface film on the electrodes of the specimen holder, $\mathrm{D}_{\mathrm{HF}}$, and those of the bridge standard capacitor, $\mathrm{D}_{2 \mathrm{~F}}$.

The dissipation factor that would be indicated by the bridge under these conditions, $D_{M}=D_{H F}-D_{2 F}$, is also plotted. These are plotted against $\Delta C_{2}$ (see text), assuming an initial value of $C_{2}$ of $20.000 \mathrm{pf}$.

${ }^{6}$ It seems reasonable to assume that a surface film would be uniformly distributed over the surface. Thus, the conductance of the film would be proportional to the active electrode area. The capacitance is also proportional to the tional to the active electrode area. The capacitance is also proportional to the
active electrode area. Therefore the resistance $R_{2} S F$ is inversely proportional to the capacitance.

Near the edges of the capacitor places, where fringing occurs, this proportionality would not hold exactly. However, results show that the nonlinearity is negligible in the present case, as might be expected from the geometry of the capacitor used.

7 This assumes that the equivalent series resistance correspon ding to the surface film losses is independent of the electric field. Tests at various voltages applied to the bridge yielded the same results, thus making this assumption appear reasonable for this case. 
Let us now consider the effect of the series resistance of the leads, switches, or other circuit components used to connect the specimen holder into the measuring circuit. A component of dissipation factor, which we shall designate $D_{H L}$, would result. This dissipation factor would behave in the same manner as $D_{H F}$, because it is of the same form:

$$
D_{H L}=\omega R_{H S L} \Delta C_{2} \text {. }
$$

Figure 5 shows a plot of $D_{H L}, D_{H F}$, and the total dissipation factor of the holder $D_{H T}$.

$$
D_{H T}=D_{H L}+D_{H F} .
$$

Assuming the same dissipation factor for the bridge standard capacitor as in the previous case, $D_{2 F}$, and plotting $D_{M}=D_{H T}-D_{2 F}$, we have the results plotted in figure 5 . We see that the slope of the $D_{M}$ plot is the same as that of $D_{H T}$, with negative intercept equal in magnitude to $D_{2 F}$.

Let us now consider the effect of series resistance in the leads connecting the bridge standard capacitor $C_{2}$ to the measuring circuit. From eq (5.3), we see that there are two terms involving this resistance: $2 \omega R_{2 S L} C_{2}^{\prime}$ and $\omega R_{2 S L} \Delta C_{2}$. Let these be designated $D_{2 L a}$ and $D_{2 L b}$ respectively. These terms plot as shown in figure 6 . Also plotted is $D_{2 T}$, the total dissipation factor of $C_{2}$.

$$
D_{2 T}=D_{2 F}+D_{2 L a}+D_{2 L b} .
$$

If we plot also the dissipation factor of the specimen holder, $D_{H T}$, and $D_{M}$, the results are as shown in figure 6. Once again we see that $D_{M}$ plots as a straight line, with a negative intercept equal to the value of $D_{2 T}$ at $\Delta C=0$. The slope of the plot of $D_{M}$ is now different from that of the plot of $D_{H}$, because of the fact that $D_{2 L b}$ is not constant.

Because the purpose of all these hypotheses is to provide a means of evaluating the various components of dissipation factor and to separate the dissipation factor of the specimen holder from that of the bridge standard capacitor, let us suppose that we

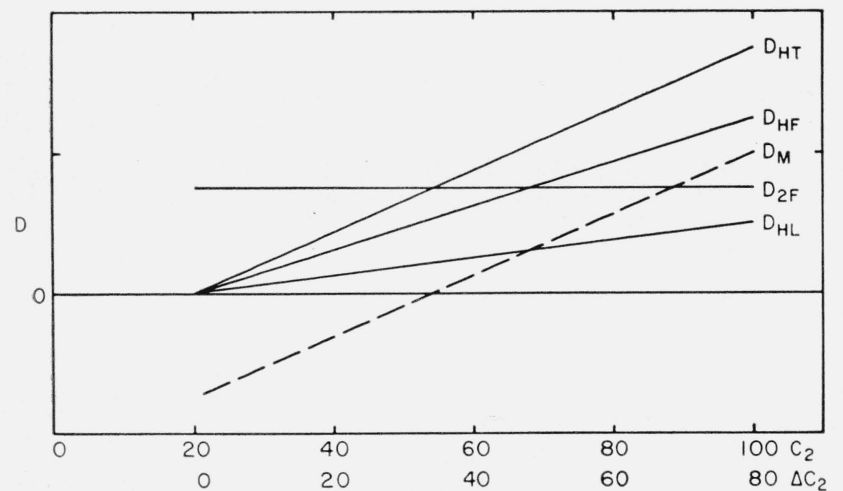

FIgURE 5. A plot similar to figure 4, but including now a component of dissipation factor due to series resistance in the leads to the specimen holder, $\mathrm{D}_{\mathrm{HL}}$.

The total dissipation factor of the holder, $D_{H T}=D_{H F}+D_{H L}$, i s plotted, and now $D_{M}=D_{H T}-D_{2 F}$

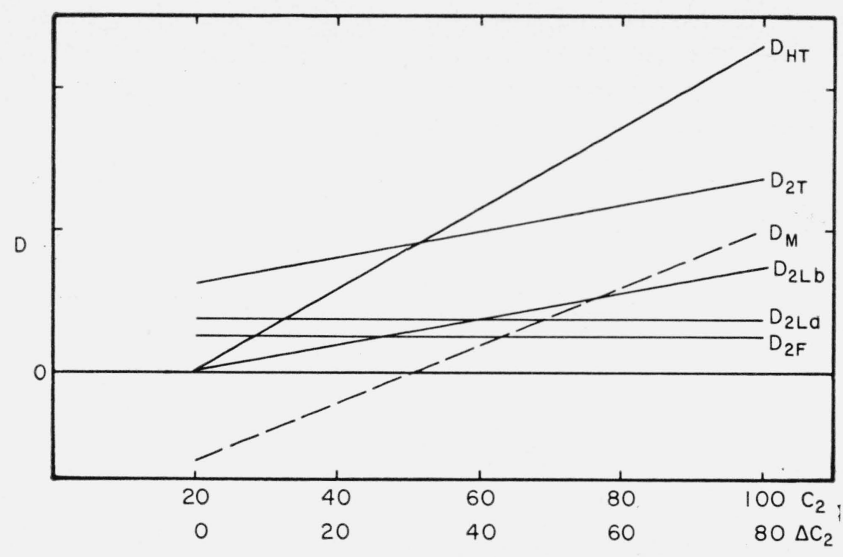

Figure 6. A plot similar to figure 5, including components of dissipation factor due to series resistance in the leads to $\mathrm{C}_{2}$, the bridge capacitor.

As discussed in the text, there are two such components, one constant with capacitance, the other proportional to $\Delta C$. $D_{2 T}$ is the sum of all components of the dissipation factor of $C_{2}$.

have just made a set of measurements that yielded the plot ${ }^{8}$ of $D_{M}$ seen in figure 6 . The slope of this curve will be the same as that of the plot of $D_{H}$ only if the slope of $D_{2 T}$ is zero. To determine the slope of $D_{2 T}$, we can make another set of measurements starting from a different $C_{2}{ }^{\prime}$. This would change the value of $D_{2 L a}$, and would permit an evaluation of this component of dissipation factor. Another revealing test would be a set of measurements at different frequencies. It can be seen that such measurements would permit the separation of dissipation factor components due to series resistance, in either the bridge standard capacitor leads or the holder leads. The behavior of the slope and intercept of the plot of $D_{M}$ would indicate the presence or absence of appreciable resistance in the leads of the bridge standard capacitor.

We have now dealt with losses in a surface film, and losses due to series resistance. Let us turn our attention to losses due to a fixed parallel conductance.

If a fixed parallel conductance occurs in the branch containing the bridge standard capacitor, it is readily apparent from eq (5.1) that it will not make any contribution to the apparent dissipation factor of the unknown being measured, for if $G_{2}{ }^{\prime}=G_{2}{ }^{\prime \prime}$, the correction term is zero.

If, however, there is a fixed conductance in parallel with the specimen holder, it will contribute a component of dissipation factor that varies with capacitance in the manner shown by the plot labelled $D_{H P}$ in figure 7 . When this is added to the other components of dissipation factor of the holder, the sum is $D_{H T}$, plotted in figure 7 . Subtracting from this $D_{2 T}$, the total dissipation factor of $C_{2}$, we have $D_{M}$, also plotted in figure 7 . It is evident that this plot behaves in a manner quite different from those previously considered, and a plot of measured values

\footnotetext{
8 Combining equations $5.2,5.3,5.4,5.5,5.6$ and 5.7 , we can write the collected expression for $D_{m}$ :

$$
D_{M}=\underbrace{\omega R_{H S L} \Delta C_{2}+\omega R_{H S F} \Delta C_{2}}_{D_{H}}-\underbrace{\omega R_{2 S F^{\prime}} C_{2}^{\prime}-2 \omega R_{2 S L} C_{2}^{\prime}-\omega R_{2 S L} \Delta C_{2}}_{D_{2}} .
$$
}




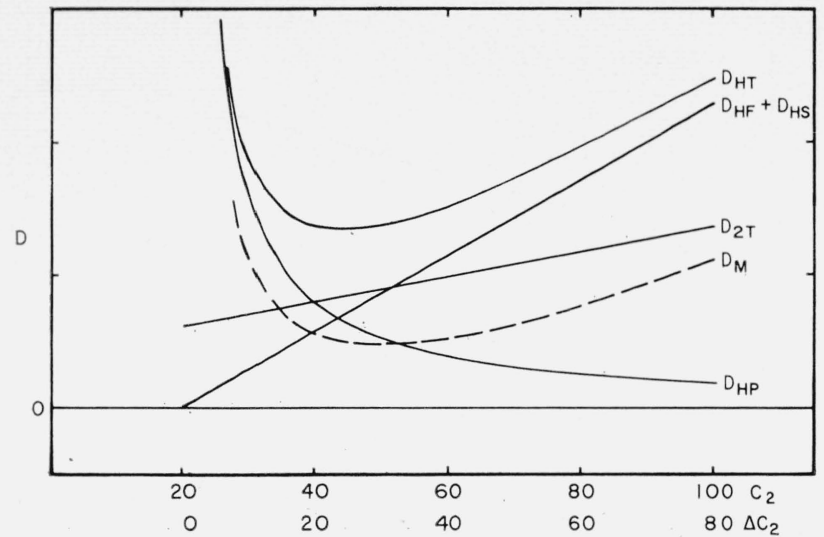

Figure 7. A plot similar to figure 6 , including a dissipation factor component due to a fixed parallel conductance in the holder, $\mathrm{D}_{\mathrm{HP}}$.

A similar conductance in $C_{2}$ would produce no effect in the measurements (see text).

of $D_{M}$ would readily reveal such a parallel conductance. In practice, such a conductance is not to be expected in the present set of circumstances, and actual measurements confirmed the absence of parallel conductance.

\subsection{Results of Measurements}

From the foregoing discussion, it is evident that we have available a means of evaluating the dissipation factor of the empty specimen holder, and also that of the bridge standard capacitor, $C_{2}$. It is only necessary to use the Schering bridge to measure the apparent dissipation factor, $D_{M}$, at several values of $C_{H}$, the capacitance of the specimen holder, recalling that (footnote 3$) C_{H} \cong \Delta C_{2}$.

Measurements were made of the losses of the new specimen holder, known as Holder No. 4, using General Radio Co. Type 722-DS29, Serial 7128 as the standard capacitor.

The measurements were made during the winter months, with room relative humidity ranging between 10 percent and 40 percent, mostly at about 20 percent.

The bridge standard capacitor, $C_{2}$, was maintained in an enclosure dried by a stream of dry nitrogen. This nitrogen stream was dried by bubbling it through concentrated sulfuric acid, thence passing it through a drying column containing calcium chloride or calcium sulfate, and finally through a dry cotton filter.

The effect of relative humidity on the dissipation factor of the holder was of considerable importance, because the holder was constructed to measure the losses in low-loss materials under different conditions of humidity, principally very dry and 52 percent relative humidity, in order to compare the results. Therefore, measurements were made on the empty holder under two conditions of relative humidity. The holder was enclosed in a chamber which was very thoroughly dried by exposing therein a generous quantity of $\mathrm{P}_{2} \mathrm{O}_{5}$, which held the relative humidity well below $1 \frac{1}{2}$ percent, which was the lower limit of the relative humidity measuring apparatus used. By exposing within this chamber a saturated solution of $\mathrm{Mg}\left(\mathrm{NO}_{3}\right)_{2}$, the relative humidity could be maintained very close to 52 percent.

Note that the controlled atmosphere applies to the specimen holder only, and not to the bridge standard capacitor, which for all measurements was dried by a stream of dry nitrogen.

Two typical sets of measurements are presented in figure 8 , for the purpose of showing that a straight line provides a reasonable fit for all the data. There is no pronounced tendency to exhibit a curve, such as the plot of $D_{M}$ in figure 7 , thus indicating that there is no appreciable parallel conductance in the holder. Note that the dissipation factors are all very small, and the slope is also small. It is not profitable to try to place any interpretation on the small digressions from the straight line, because the resolution of the apparatus is not great enough to permit more accurate measurements of such small dissipation factors. The resolution is much better at high capacitances than at the low end. Equation (5.1) gives, neglecting the correction term,

$$
D_{H}=\frac{\omega R_{3} C_{2}^{\prime \prime} \Delta C_{3}}{\Delta C_{2}},
$$

from which it is seen that as the measured dissipation factor becomes very small, $\Delta C_{3}$ becomes very small. Since it is very difficult to determine very small differences in relatively large quantities, the method gives rather poor resolution at the lower values of dissipation factor, and this effect is heightened by the fact that the smallest dissipation factors occur when $\Delta C_{2}$ is smallest. For these reasons, no measurements at $\Delta C_{2}$ less than $20 \mathrm{pf}$ are included.

Most of the dielectric specimens for which this new holder was designed will have capacitances of 5 to 50 pf. Referring again to figure 8, notice that in this range the dissipation factor does not vary more than about $4 \times 10^{-6}$, or in terms of the loss angle, ${ }^{9}$ about 4 microradians ( $\mu$ radians).

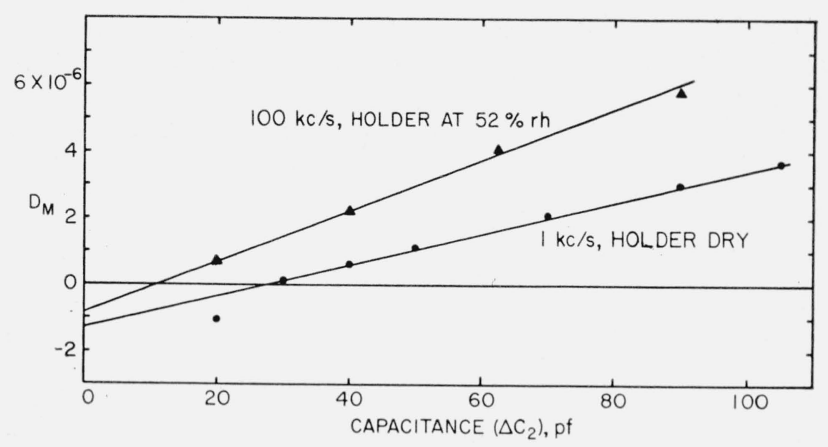

FIGURE 8. Typical results of actual measurement of dissipation factor of the holder under two widely different conditions, showing that straight-line plots adequately represent the data, and that the dissipation foctors are small.

The dissipation factor, $D$, is the tangent of the loss angle. $D=\tan \delta$. For small angles $\delta \cong \tan \delta$, and often the term loss angle, expressed in $\mu$ radians is used. 
The magnitudes of the intercepts of the two sets of measurements are less than $2 \mu$ radians. In the light of the preceding analysis, this can be interpreted to show that the sum of the components of loss angle caused by the surface film on the electrodes of the bridge standard capacitor, $C_{2}$, and that caused by series resistance in the leads to $C_{2}$ is less than 2 $\mu$ radians at $\Delta C_{2}=0$. This sets a limit to the slope of the plot of the total dissipation factor of $C_{2}$ versus $\Delta C_{2}$, as is evident from examination of eq (5.3). For convenience this expression is rewritten here:

$$
D_{H}=D_{M}+2 \omega R_{2 S L} C_{2}^{\prime}+\omega R_{2 S L} \Delta C_{2}+\omega C_{2}^{\prime} R_{2 S F^{\prime}} .
$$

Note that the third term on the right is the same as the second term on the right when $\Delta C_{2}=2 C_{2}{ }^{\prime}$. Thus if the loss angle of the bridge standard capacitor is less than $2 \mu$ radians at $\Delta C_{2}=0$, it must be less than 4 $\mu$ radians at $\Delta C_{2}=2 C_{2}{ }^{\prime}=40.000 \mathrm{pf}$. Note also that this is true at the highest frequency used, $100 \mathrm{kc} / \mathrm{s}$. Even if the third term on the right were at its maximum, almost $2 \mu$ radians, at this frequency, it would be only one-tenth of this or $0.2 \mu \mathrm{radian}$ at $10 \mathrm{kc} / \mathrm{s}$, and would be no more than $0.02 \mu$ radian at $1 \mathrm{kc} / \mathrm{s}$.

To sum up this line of reasoning, we have proven that at frequencies up to $100 \mathrm{kc} / \mathrm{s}$, and at specimen holder capacitances up to $40 \mathrm{pf}\left(C_{H} \cong \Delta C_{2}\right)$, the maximum error in the determination of the loss angle of the empty specimen holder due to neglected series resistance in the leads to the bridge standard capacitor is no greater than $2 \mu$ radians. Other tests show that the actual error due to this cause is much smaller than this maximum. Such tests include measurements made at various frequencies, from other initial values of $C_{2}$, and on actual specimens of very low loss materials, such as polyethylene.

Our early measurements showed a much greater slope for $D_{M}$ at $100 \mathrm{kc} / \mathrm{s}$. Examination showed this loss to be attributable to the series resistance of certain leads used at that time. The offending cable was removed and replaced with a type with much lower resistance, and a great improvement was observed. The effect of the residual series resistance in the leads to the specimen holder are still observable in figure 9 , where the results of measurements at frequencies of $100 \mathrm{c} / \mathrm{s}, 1 \mathrm{kc} / \mathrm{s}, 10 \mathrm{kc} / \mathrm{s}$, and $100 \mathrm{kc} / \mathrm{s}$ are shown together. Note that the slope of the $100 \mathrm{kc} / \mathrm{s}$ plot is greater than the other slopes. The results shown in figure 9 show that at all measured frequencies except $100 \mathrm{c} / \mathrm{s}$, the intercept is about $1 \mu$ radian or less. This confirms and emphasizes that the series resistance in in the leads to $C_{2}$ is low.

The data presented in figure 9 were obtained with the specimen holder maintained in a very dry atmosphere. In figure 10 we see the results obtained when this atmosphere had a relative humidity of about 52 percent. Note that in both these cases, the bridge standard capacitor, $C_{2}$, was maintained as dry as could be attained with a stream of dry nitrogen flowing through the enclosure containing it. This means that losses in $C_{2}$, indicated principally by the intercepts, should remain nearly unchanged for the two sets of data. The higher relative humidity might be expected to increase

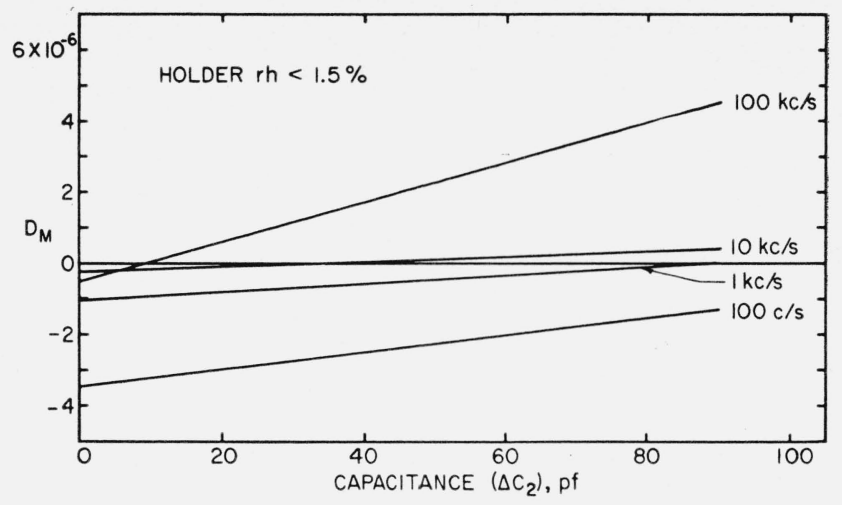

Figure 9. Results obtained at $100 \mathrm{c} / \mathrm{s}, 1 \mathrm{kc} / \mathrm{s}, 10 \mathrm{kc} / \mathrm{s}$, and $100 \mathrm{kc} / \mathrm{s}$, with the holder maintained in a dry (relative humidity less than 1.5 percent) atmosphere.

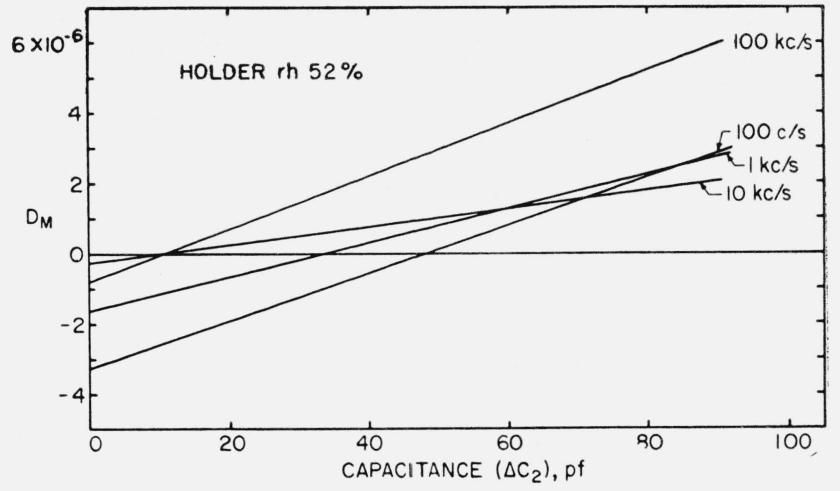

Figure 10. Results obtained at $100 \mathrm{c} / \mathrm{s}, 1 \mathrm{kc} / \mathrm{s}, 10 \mathrm{kc} / \mathrm{s}$, and 100 $\mathrm{kc} / \mathrm{s}$, with the holder maintained in an atmosphere of 52 percent relative humidity.

the loss in the surface film on the electrodes of the holder. This would be evidenced by an increased slope for $D_{M}$ plots at the higher relative humidity.

Comparison of figures 9 and 10 shows that actual measurements agree with the behavior anticipated. The intercepts, within experimental error, are the same for both cases, while the slopes are noticeably greater for the higher humidity case.

The agreement of the intercepts is even more evident in figure 11, where values of $D_{2}$ obtained under the two different conditions of relative humidity surrounding the holder are plotted together. The agreement is satisfactory, and the curve drawn through the average values shows the variation of $D_{2}$ as a function of frequency. The higher dissipation factor at the lower frequencies is evident here, as well as a very slight increase at the high end of the frequency scale. This latter is probably due to the residual series resistance of the leads to the bridge standard capacitor, $C_{2}$.

Early in the course of this investigation it was discovered that the apparent loss of the empty specimen holder was profoundly affected by the presence of small amounts of foreign materials on the electrode surfaces. It was necessary to keep the electrodes scrupulously clean, by occasionally cleaning them with distilled water followed by petroleum ether. It was found that electrodes 


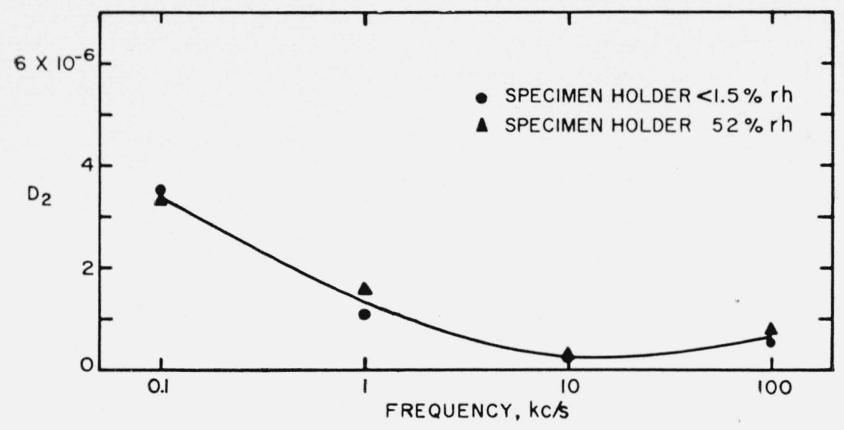

FIGURE 11. The dissipation factor, $\mathrm{D}_{2}$, of the bridge standard capacitor, $\mathrm{C}_{2}$, plotted as a function of frequency.

Each value is obtained from the intercept of one of the preceding plots, one set from the dry holder plots of figure 9 , the other set from the 52 percent relative
humidity holder plots of figure 10 . Note that $C_{2}$ was maintained in an enhumidity holder plots of figure 10 . Note that $C_{2}$ was maintained in an
closure dried by a stream of dry nitrogen for all measurements (see text).

applied to dielectric specimens must be very clean, to avoid carrying dust particles, etc. to the holder electrodes. The care previously used in the handling of specimens was found to be inadequate, and new precautions were initiated, especially in the case of low loss materials, where a loss contribution due to foreign matter would be a greater percentage of the whole loss.

\subsection{Correction for Holder Losses in Dielectric Measurements}

After the values of the dissipation factors of the bridge standard capacitor and the empty specimen holder have been determined, the next problem is how to use them properly to correct the measured dissipation factor of a dielectric specimen.

In all cases, it is clear that $D_{2}$, the dissipation factor of the bridge standard capacitor, should be added to the measured value of dissipation factor of the specimen, $D_{M}$.

If the dielectric specimen has no contact electrodes applied to it, and is larger than the guarded electrode, it is evident that the measured dissipation factor will consist of two components, one due to losses in the dielectric material, the other due to losses in the holder. The latter component, which can be determined by the method described herein, can be subtracted from the measured value to yield the dissipation factor of the dielectric material itself, taking into account the fact that the dielectric constant of the specimen multiplies the effect of $D_{H}$. The correct relation to use in this case is:

where

$$
D_{x}=D_{M}-\epsilon_{x}^{\prime} D_{H}+D_{2},
$$

\section{$D_{x}=$ dissipation factor of the specimen}

and

$$
\boldsymbol{\epsilon}_{x}^{\prime}=\text { dielectric constant of the specimen. }
$$

In the case of a specimen with contact electrodes applied, this question must be answered: does the surface film remain intact, or is electrical contact made directly from the conducting contact electrode to the conducting substrate of the holder electrode material? If the latter is true, then the dielectric film is in effect short-circuited, and does not affect the measured dissipation factor. The problem is then shifted to that of determining what losses occur in the contact electrode material, in the interface of the contact electrode and the dielectric specimen, and in the material serving as adhesive, if any. This is a separate matter, and will not be further treated here.

If the specimen is smaller than the guarded electrode, so that some air capacitance is included in the measurement, the problem can be resolved into two parts, one dealing with the portion involving air capacitance in the space surrounding the specimen, the other dealing with the portion of the volume between the electrodes that is occupied by the dielectric specimen, taking into account its contact electrodes, if any.

Admittedly, some uncertainties arise here with respect to what happens in the case where contact electrodes are used. However, the fact that the surface film contributes (in this case) such a small component of loss, saves one from commiting a grave error, and it is probably best to make reasonable assumptions and proceed, secure in the knowledge that at worst the error will not exceed a few microradians.

\subsection{Implications for Further Investigation}

A second specimen holder is under construction, quite similar in basic design to the one described herein, except that provision is being made to make the electrodes interchangeable, and several sets of electrodes are in preparation. These electrodes are made of various materials, and will be used to determine which material yields lowest losses in surface layers. In addition to measuring several solid electrodes, it is intended that platings of various kinds will be applied to some of the electrode sets, and the losses in the surfaces measured.

\section{Summary and Conclusions}

A guard-ring micrometer-electrode specimen holder, especially designed for use for precision measurements of the dielectric properties of dielectric reference standard materials, has been constructed and evaluated. A technique has been devised for determining the spacing between the electrodes to \pm 1 $\mu$, using the micrometer.

It was found that resistance in cables often used to connect the components of a bridge can produce losses which are appreciable, and unless a correction is made for such losses, they can produce errors in the values of the dissipation factor of the holder, and also that of the bridge standard capacitor. A preferable method is to keep these resistances as low as possible by proper choice of cable type.

It was found also that minute quantities of foreign matter (such as dust or grease) on the holder electrodes will contribute an appreciable increment to the dissipation factor. It is necessary to be very careful to keep the electrodes scrupulously clean when low-loss materials are being measured. 
The dissipation factor of the carefully cleaned holder has been measured, and it has been concluded that at frequencies in the range $100 \mathrm{c} / \mathrm{s}$ to $100 \mathrm{kc} / \mathrm{s}$, almost all of the loss is in the surface film on the Type 304 stainless steel electrodes. The value of this dissipation factor was found to be directly proportional to the capacitance of the holder, and therefore inversely proportional to the separation of the electrodes. The relative humidity of the atmosphere surrounding the holder had an effect on the surface losses. The dissipation factor varied from about $1 \times 10^{-8}$ per picofarad at $1 \mathrm{kc} / \mathrm{s}$ in a very dry atmosphere, to about $7 \times 10^{-8}$ per picofarad at $100 \mathrm{kc} / \mathrm{s}$ with an atmosphere of about 52 percent relative humidity.

Knowledge of the values of these empty specimen holder losses is very useful for making corrections to the measured loss of dielectric materials with low losses, where the loss contribution of the electrode surface may be an appreciable fraction of the total loss.

Measurements made by the substitution method with a Schering bridge should also be corrected for the error due to the dissipation factor of the bridge standard capacitor. The method used yielded the value of this correction. The loss in a General Radio Type 722-DS29 variable air capacitor was concluded to be almost entirely in the electrode surfaces, and it was confirmed that the dissipation factor at any single frequency is almost constant over the entire capacitance range. When the capacitor was maintained in an enclosure dried by a stream of dry nitrogen, the dissipation factor was found to range from less than $0.5 \times 10^{-6}$ at $10 \mathrm{kc} / \mathrm{s}$, to about $3.5 \times 10^{-6}$ at $100 \mathrm{c} / \mathrm{s}$.

With this new specimen holder, it is possible to measure the spacing of the electrodes with about the same accuracy as that with which the thickness of the specimens can be determined. With the application of corrections determined by the methods described in this paper, the holder can be used to measure the loss angle of low-loss materials to within a few microradians.

\section{Appendix}

The exact expression for $C_{H}$ as a function of the Schering bridge parameters is

$C_{H}=C_{2}^{\prime \prime} \frac{G_{3}^{\prime \prime} G_{3}^{\prime}+\omega^{2} C_{3}^{\prime} C_{3}^{\prime \prime}}{G_{3}^{\prime \prime 2}+\omega^{2} C_{3}^{\prime \prime 2}}-C_{2}^{\prime}+G_{2}^{\prime \prime} \frac{G_{3}^{\prime \prime} C_{3}^{\prime}-G_{3}^{\prime} C_{3}^{\prime \prime}}{G_{3}^{\prime \prime 2}+\omega^{2} C_{3}^{\prime \prime 2}}$.

The expression for $D_{H}$ is

$$
\begin{aligned}
D_{H} & =\frac{\omega C_{2}^{\prime \prime}}{C_{3}^{\prime \prime} C_{H}}\left[C_{3}^{\prime \prime}\left(\frac{G_{3}^{\prime \prime} G_{3}^{\prime}+\omega^{2} C_{3}^{\prime} C_{3}^{\prime \prime}}{G_{3}^{\prime \prime 2}+\omega^{2} C_{3}^{\prime \prime 2}}\right)-C_{3}^{\prime}\right] \\
& +\frac{G_{2}^{\prime \prime}}{\omega C_{H}}\left[\frac{G_{3}^{\prime}}{G_{3}^{\prime \prime}}-\frac{\omega^{2} C_{3}^{\prime \prime}}{G_{3}^{\prime \prime}}\left(\frac{G_{3}^{\prime} C_{3}^{\prime \prime}-G_{3}^{\prime \prime} C_{3}^{\prime}}{G_{3}^{\prime \prime 2}+\omega^{2} C_{3}^{\prime \prime 2}}\right)\right]-\frac{G_{2}^{\prime}}{\omega C_{H}} .
\end{aligned}
$$

where

$D_{H}=$ dissipation factor of the specimen holder and, in general, any specimen contained therein;

$C_{2}^{\prime}=$ initial value of $C_{2}$, with switch 1 , figure 3 , in position $\mathrm{A}$;

$C_{2}^{\prime \prime}=$ final value of $C_{2}$, with switch 1 in position $\mathrm{B}$;

$\Delta C_{2}=C_{2}^{\prime \prime}-C_{2}^{\prime}$;

$C_{3}^{\prime}=$ initial value of $C_{3}$, with switch 1 in position A;

$C_{3}^{\prime \prime}=$ final value of $C_{3}$, with switch 1 in position $\mathrm{B}$;

$\Delta C_{3}=C_{3}{ }^{\prime \prime}-C_{3}{ }^{\prime}$;

$G_{2}^{\prime}=$ initial value of equivalent parallel conductance associated with $C_{2}$;

$G_{2}^{\prime \prime}=$ final value of equivalent parallel conductance associated with $C_{2}$;

$G_{3}^{\prime}=$ initial value of equivalent parallel conductance associated with $C_{3}$; and

$G_{3}^{\prime \prime}=$ final value of equivalent parallel conductance associated with $C_{3}$.

Since $G_{3}$ is principally the conductance of a fixed precision resistor of not more than $10^{5} \mathrm{ohms}$, it is safe to assume that

$$
G_{3}^{\prime}=G_{3}^{\prime \prime}=G_{3} .
$$

We can now write

$$
\begin{aligned}
D_{H}=\frac{\omega C_{2}^{\prime \prime}}{G_{3} C_{H}}[ & \left.C_{3}^{\prime \prime}\left(\frac{G_{3}^{2}+\omega^{2} C_{3}^{\prime} C_{3}^{\prime \prime}}{G_{3}^{2}+\omega^{2} C_{3}^{\prime \prime 2}}\right)-C_{3}^{\prime}\right] \\
& +\frac{G_{2}^{\prime \prime}}{\omega C_{H}}\left[1-\frac{\omega^{2} C_{3}^{\prime \prime}\left(C_{3}^{\prime \prime}-C_{3}^{\prime}\right)}{G_{3}^{2}+\omega^{2} C_{3}^{\prime \prime 2}}\right]-\frac{G_{2}^{\prime}}{\omega C_{H}} .
\end{aligned}
$$

For the Schering bridge used, $\omega / G_{3}$ was held constant at $2 \pi \times 10^{7}, C_{3}^{\prime \prime}$ never exceeded $1100 \mathrm{pf}$, and $C_{3}^{\prime \prime}-C_{3}^{\prime}$ never exceeded 1000 pf. Under these conditions we may write

$D_{H}=\frac{\omega C_{2}^{\prime \prime}}{G_{3} C_{H}}\left[C_{3}^{\prime \prime}\left(\frac{G_{3}^{2}+\omega^{2} C_{3}^{\prime} C_{3}^{\prime \prime}}{G_{3}^{2}+\omega^{2} C_{3}^{\prime \prime 2}}\right)-C_{3}^{\prime}\right]+\frac{G_{2}^{\prime \prime}}{\omega C_{H}^{\prime}}-\frac{G_{2}^{\prime}}{\omega C_{H}^{\prime}}$,

with no appreciable error in $D_{H}$. And

$$
D_{H}=\frac{\omega C_{2}^{\prime \prime}}{G_{3} C_{H}}\left(C_{3}^{\prime \prime}-C_{3}^{\prime}\right)+\frac{G_{2}^{\prime \prime}-G_{2}^{\prime}}{\omega C_{H}}
$$

will give no more than 0.5 percent error in $D_{H}$. For less extreme values of $C_{3}^{\prime \prime}$, the error is less. The present work involved very low $D_{H}$, and therefore very small $C_{3}^{\prime \prime}-C_{3}^{\prime}$, resulting in wholly negligible errors due to terms omitted from eq (7.5).

The first term on the right in eq (7.5) may be expressed as

yielding

$$
\frac{\omega R_{3} C_{2}^{\prime \prime}}{C_{H}}\left(C_{3}^{\prime \prime}-C_{3}^{\prime}\right)
$$

$$
D_{H}=\frac{\omega R_{3} C_{2}^{\prime \prime} \Delta C_{3}}{C_{H}}+\frac{G_{2}^{\prime \prime}-G_{2}^{\prime}}{\omega C_{H}}
$$


In eqs (7.2) through (7.6), the exact expression for $C_{H}$ given by eq (7.1) may be used. For the present work, however, $C_{3}^{\prime}$ was about $100 \mathrm{pf}$ and $C_{3}^{\prime \prime}$ was less than $150 \mathrm{pf}$, yielding an error in $C_{H}$ less than 0.003 percent if the correction terms in eq (7.1) are neglected. Therefore, we may with negligible further error write $\Delta C_{2}$ for $C_{H}$ in the preceding equations. Thus our expression for $D_{H}$ becomes

$$
D_{H}=\frac{\omega R_{3} C_{2}^{\prime \prime} \Delta C_{3}}{\Delta C_{2}}+\frac{G_{2}^{\prime \prime}-G_{2}^{\prime}}{\omega \Delta C_{2}}
$$

The last term in eq (7.7) is a correction term necessary if $G_{2}^{\prime \prime} \neq G_{2}^{\prime}$. If this correction term is ignored, or is negligible, $D_{H}$ is the term $\omega R_{3} C_{2}{ }^{\prime \prime} \Delta C_{3} / \Delta C_{2}$ which we will call $D_{M}$, the measured or uncorrected value of $D_{H}$. Then

$$
D_{H}=D_{M}+\frac{G_{2}^{\prime \prime}-G_{2}^{\prime}}{\omega \Delta C_{2}} .
$$

For present purposes it will be useful to express this in terms of equivalent series resistances.

In parallel notation $D=G_{P} / \omega C_{P}$, where

$$
\begin{aligned}
& G_{P}=\text { equivalent parallel conductance } \\
& C_{P}=\text { equivalent parallel capacitance. }
\end{aligned}
$$

Therefore, let us write

$\frac{G_{2}^{\prime \prime}-G_{2}^{\prime}}{\omega \Delta C_{2}}=\frac{C_{2}^{\prime \prime} G_{2}^{\prime \prime}}{\Delta C_{2} \omega C_{2}^{\prime \prime}}-\frac{C_{2}^{\prime} G_{2}^{\prime}}{\Delta C_{2} \omega C_{2}^{\prime}}=\frac{C_{2}^{\prime \prime}}{\Delta C_{2}^{\prime}} D_{2}^{\prime \prime}-\frac{C_{2}^{\prime}}{\Delta C_{2}} D_{2}^{\prime}$.

In series notation, $D=\omega R_{S} C_{S}$,

where

$$
\begin{aligned}
& R_{S}=\text { equivalent series resistance } \\
& C_{S}=\text { equivalent series capacitance. }
\end{aligned}
$$

Therefore if we let

$C_{2 S}^{\prime}=$ series equivalent of $C_{2}^{\prime}$

$C_{2 S}^{\prime \prime}=$ series equivalent of $C_{2}^{\prime \prime}$

$R_{2 S}^{\prime \prime}=$ equivalent series resistance corresponding to $G_{2}^{\prime}$ $R_{2 S}^{\prime \prime}=$ equivalent series resistance corresponding to $G_{2}^{\prime \prime}$

then

$\frac{C_{2}^{\prime \prime}}{\Delta C_{2}} D_{2}^{\prime \prime}-\frac{C_{2}^{\prime}}{\Delta C_{2}} D_{2}^{\prime}=\frac{C_{2}^{\prime \prime}}{\Delta C_{2}} \omega R_{2 S}^{\prime \prime} C_{2 S}^{\prime \prime}-\frac{C_{2}^{\prime}}{\Delta C_{2}} \omega R_{2 S}^{\prime} C_{2 S}^{\prime}$.

Because the dissipation factors involved in this work are very small, the series equivalent capacitance is very nearly the same as the parallel capacitance. That is

Therefore

$$
C_{2 S}^{\prime} \cong C_{2}^{\prime} \text {, and } C_{2 S}^{\prime \prime} \cong C_{2}^{\prime \prime}
$$

$\frac{C_{2}^{\prime \prime}}{\Delta C_{2}} \omega R_{2 S}^{\prime \prime} C_{2 S}^{\prime \prime}-\frac{C_{2}^{\prime}}{\Delta C_{2}} \omega R_{2 S}^{\prime} C_{2 S}^{\prime}=\frac{C_{2}^{\prime \prime 2} \omega R_{2 S}^{\prime \prime}}{\Delta C_{2}}-\frac{C_{2}^{\prime 2} \omega R_{2 S}^{\prime}}{\Delta C_{2}}$.
Let us now consider the series resistance $R_{2 S}$ to be composed of two components: (1) a series resistance term $R_{2 S L}$ representing series ohmic resistance in leads, connections etc. and (2) a term $R_{2 S F}$ representing the series equivalent resistance of a surface film on the electrodes. That is

$$
\begin{aligned}
& R_{2 S}^{\prime}=R_{2 S L}^{\prime}+\mathrm{R}_{2 S F}^{\prime} \\
& R_{2 S}^{\prime \prime}=R_{2 S L}^{\prime \prime}+R_{2 S F}^{\prime \prime}
\end{aligned}
$$

Because $R_{2 S L}$ represents series ohmic resistance, this term is independent of $C_{2}$. That is

and

$$
R_{2 S L}^{\prime}=R_{2 S L}^{\prime \prime}=R_{2 S L}
$$

$$
\begin{gathered}
D_{H}=D_{M}+\frac{C_{2}^{\prime \prime 2} \omega\left(R_{2 S L}+R_{2 S F}^{\prime \prime}\right)}{\Delta C_{2}}-\frac{C_{2}^{\prime 2} \omega\left(R_{2 S L}+R_{2 S F}^{\prime}\right)}{\Delta C_{2}} \\
=D_{M}+\frac{\left(C_{2}^{\prime \prime 2}-C_{2}^{\prime 2}\right) \omega R_{2 S L}}{\Delta C_{2}} \\
\quad+\frac{C_{2}^{\prime \prime 2} \omega R_{2 S F}^{\prime \prime}}{\Delta C_{2}}-\frac{C_{2}^{\prime 2} \omega R_{2 S F}^{\prime} .}{\Delta C_{2}}
\end{gathered}
$$

Because $C_{2}^{\prime \prime}=C_{2}^{\prime}+\Delta C_{2}$

$$
\begin{aligned}
D_{H}=D_{M}+\frac{\left(C_{2}^{\prime \prime 2}-C_{2}^{\prime 2}\right) \omega R_{2 S L}}{\Delta C_{2}}+\frac{\omega C_{2}^{\prime \prime} R_{2 S F}^{\prime \prime} C_{2}^{\prime}}{\Delta C_{2}} \\
+\frac{\omega C_{2}^{\prime \prime} R_{2 S F}^{\prime \prime} \Delta C_{2}}{\Delta C_{2}}-\frac{\omega C_{2}^{\prime} R_{2 S F}^{\prime} C_{2}^{\prime}}{\Delta C_{2}} .
\end{aligned}
$$

The conductance of the surface film, $1 / R_{2 S F}$, is proportional to the active area of the capacitor plates, which may be expressed

$$
1 / R_{2 S F}=\alpha A \text {. }
$$

From this

$$
R_{2 S F}=1 / \alpha A \text {. }
$$

The capacitance $C_{2}$ is also proportional to the active area of the capacitor plates, or

$$
C_{2}=\beta A \text {. }
$$

The product $R_{2 S F} C_{2}$ is now seen to be a constant because

$$
R_{2 S F} C_{2}=\frac{\beta A}{\alpha A}=\frac{\beta}{\alpha},
$$

the ratio of two proportionality constants.

From this we see that

Therefore

$$
R_{2 S F}^{\prime \prime} C_{2}^{\prime \prime}=R_{2 S F}^{\prime} C_{2}^{\prime}
$$

$$
\begin{gathered}
D_{H}=D_{M}+\frac{\left(C_{2}^{\prime \prime 2}-C_{2}^{\prime 2}\right) \omega R_{2 S L}+\frac{\omega C_{2}^{\prime} R_{2 S F}^{\prime} C_{2}^{\prime}}{\Delta C_{2}}}{\Delta C_{2}} \\
-\frac{\omega C_{2}^{\prime} R_{2 S F}^{\prime} C_{2}^{\prime}}{\Delta C_{2}}+\omega C_{2}^{\prime} R_{2 S F^{*}}^{\prime} \\
D_{H}=D_{M}+\frac{\left(C_{2}^{\prime \prime 2}-C_{2}^{\prime 2}\right) \omega R_{2 S L}}{\Delta C_{2}}+\omega C_{2}^{\prime} R_{2 S F^{*}}^{\prime}
\end{gathered}
$$


Attacking now the second term on the right, we can write

$$
D_{H}=D_{M}+\frac{\left(C_{2}^{\prime \prime}-C_{2}^{\prime}\right)\left(C_{2}^{\prime \prime}+C_{2}^{\prime}\right) \omega R_{2 S L}}{\Delta C_{2}}+\omega C_{2}^{\prime} R_{2 S F^{*}}^{\prime}
$$

Since $C_{2}^{\prime \prime}-C_{2}^{\prime}=\Delta C_{2}$

$$
D_{H}=D_{M}+\left(C_{2}^{\prime \prime}+C_{2}^{\prime}\right) \omega R_{2 S L}+\omega C_{2}^{\prime} R_{2 S F}^{\prime},
$$

and because $C_{2}^{\prime \prime}=\Delta C_{2}+C_{2}^{\prime}$,

$$
D_{H}=D_{M}+2 \omega C_{2}^{\prime} R_{2 S L}+\omega \Delta C_{2} R_{2 S L}+\omega C_{2}^{\prime} R_{2 S F}^{\prime} .
$$

The authors wish to acknowledge the helpful comments and suggestions of John D. Hoffman and Robert D. Cutkosky, and a contribution to the analysis of the losses by Donald J. Scheiber.

(Paper 65C2-61)

\section{References}

[1] A. H. Scott and W. P. Harris, A study of materials for their suitability as standard dielectric reference materials, 1959 Annual Report, Conference on Electrical Insulation, Nat. Acad. Sci.-Nat. Research Council, Publ. 756, 1, (1960)

[2] Methods of test for A-C capacitance, dielectric constant, and loss characteristics of electrical insulating materials, Am. Soc. Testing Materials, Standards, Pt. 9, 1959 Supplement, D $150-59$ T, p. 154 (1959).

[3] J. C. Balsbaugh and P. H. Moon, A bridge for precision power factor measurements on small oil samples, Trans. Am. Inst. Elec. Engrs. 52, 528 (1933).

[4] J. C. Balsbaugh and A. Herzenberg, Comprehensive theory of a power factor bridge, J. Franklin Inst. 218, 49 (1934).

[5] W. B. Kouwenhoven and E. L. Lotz, Absolute power factor of air capacitors, Trans. Am. Inst. Elec. Engrs. 57, (1938)

[6] A. V. Astin, Measurement of relative and true power factors of air capacitors, J. Research NBS, 21, 425 (1938).

[7] A. V. Astin, Nature of energy losses in air capacitors at low frequencies, J. Research NBS, 22, 673 (1939).

[8] C. G. Koops, The measurement of very small phase displacements, Philips Tech. Rev., 5, 300 (1940).

[9] W. Ch. van Geel and B. J. J. Schelen, Some properties of oxide layers produced on aluminum by electrolytic oxidation, Philips Research Reps. 12, 240 (1957).

[10] P. Winkel and D. G. de Groot, Impedance of dielectric layers, Philips Research Reps. 13, 489 (1958).

[11] A. M. Thompson, The precise measurement of small capacitances, Inst. Radio Engrs., Trans. on Instrumentation I-7, 245 (1958).

[12] Unpublished notes of R. D. Cutkosky, NBS.

[13] M. Kanno and Y. Koizumi, Determination of true loss angle of capacitors, J. Electro-tech. Lab., Japan (June 17, 1956). (In Japanese) 